\title{
Mechanical Modeling and Finite Element Analysis of Porous Cast Products*
}

\section{N. Esmaeili" ${ }^{* *}$ J.L. Alves ${ }^{* *, * * *}$ and C. Teodosiu ${ }^{* *}$}

Key words

porosity, serial sectioning, finite element modeling, aluminum die-cast, elastic properties, fatigue, fracture

\section{Introduction}

Porosity has a considerable influence on the physical properties of many mechanical properties of materials, such as elastic moduli, yield limit, strain hardening, fatigue and ductile fracture. The volume fraction of pores, as well as their average size, shape and location are generally acknowledged as affecting both the dynamic and the static properties of cast alloys intended for structural applications ${ }^{1) 33}$. Therefore, incorporation of a realistic description of three-dimensional (3D) microstructures into the micromechanical analysis of such materials is of particular interest. In a cast product, porosity is often nonuniformly distributed over the part, and hence the material properties of the casts are mostly heterogeneous.

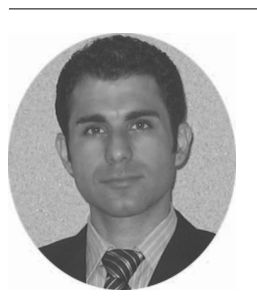

*原稿受付 平成 20 年 10 月 27 日

**VAD System Research Program, RIKEN, Japan

${ }^{* * *}$ Department of Mechanical Engineering, University of Minho, Portugal

Naghi Esmaeili K.

received a $\mathrm{Ph} . \mathrm{D}$. in mechanical engineering from Kobe University, Japan (2005). He is currently a researcher of VCAD System Research Program, RIKEN. His present researches consist of structural analysis of multimaterials, and in particular elastoplastic structural analysis of porous metallic materials.

J.L. Alves

is Assistant Professor at the Department of Mechanical Engineering of the University of Minho, Portugal. Received a Ph.D in Computational Mechanics applied to Manufacturing Technologies in 2003. Since then has developed his research activities on several institutes, namely, LPMTM-CNRS in France and RIKEN Institute in Japan. Was awarded in 2006 with the ESAFORM Scientific Prize.

Cristian Teodosiu

is laboratory head within the Volume-CAD System Research Program, at RIKEN, Japan. He was Research Director at CNRS, France and visiting professor at several universities in France, Germany and Romania. His main research fields have been the non-linear mechanics of materials and structures, and the simulation of forming processes. He received his M. S. in Civil Engineering in 1958 from the Institute of Civil Engineering, Bucharest, Romania, his M. S. in Mathematics and Physics in 1960 from the University of Bucharest, Romania and his Ph. D. in Mathematics in 1966 from the Institute of Mathematics of the Romanian Academy.
Macroporosity in metals, generally defined as porosity visible without magnification and typically of average size larger than $100 \mu \mathrm{m}$, can cause gross section loss, thus reducing the effective elastic moduli or stiffness ${ }^{4)}$. On the other hand, even if microporosity may not result in high stress concentrations and stress redistribution, it may significantly affect the fatigue resistance ${ }^{5) \sim 7)}$. Several studies have been carried out on the correlation of the porosity and mechanical properties of the cast products ${ }^{899)}$. While elastic properties of porous materials are primarily related to the volume fraction of the pores, fracture-related properties do not quantitatively correlate to the first-order average microstructural characteristics of the pores, such as volume fraction and size $\mathrm{e}^{1011)}$. This is because the fracture nucleation and path is also sensitive to secondorder characteristics, such as the extent of pore clustering and the size correlations of clustered pores.

The VCAD System Research Program ${ }^{12}$ aims at developing a methodology to perform $\mathrm{FE}$-based simulations on realistic 3D images of multi-materials, reconstructed either from a stack of microstructural serial sections or from X-ray computer tomography. In this context, VCAD offers an excellent environment for the high-resolution data acquisition, as well as for the 3D reconstruction, data resampling and denoising, mesh generation and simplification. In this study, serial sectioning has been used to generate 3D microstructural images, which are then embedded into a FE software to simulate the influence of porosity on the mechanical response of the cast aluminum product. The present paper aims at developing a methodology to perform $\mathrm{FE}$-based simulations of the micromechanical response on actual 3D microstructures. Although the methodology is developed for microstructures reconstructed from serial sections, it is equally applicable to other 3D image acquisition techniques such as X-ray computed tomography. 


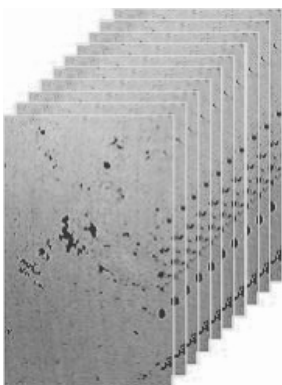

(a)

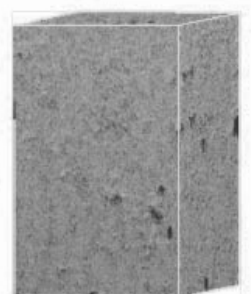

(b)

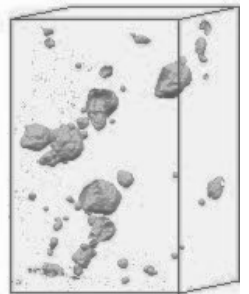

(c)
Fig. 1 (a) 2D serial sectioning images. (b) Reconstruction of a RVE. (c) Visualization of gas and shrinkage pores.

\section{Visualization and reconstruction of microstructure}

Serial sectioning is an established method for generating very accurate 3D microstructural data through the controlled repetition of sectioning technique ${ }^{13) 14)}$. Serial sectioning involves observation of an area of a metallographic plane and photographing or grabbing its digital image, removing a small amount of specimen thickness (1 $5 \mu \mathrm{m})$ by precision milling, and observing an area in exactly the same location as the first section. The image of following section is photographed, and the procedure is repeated until the whole material volume is depleted. Then, the 3D microstructure can be reconstructed from these 2D sections. In this study, the serial sectioning technique is applied for the high-resolution reconstruction of the 3D microstructure of a porous die cast aluminum alloy, thus enabling the visualization and reconstruction of detailed morphologies and spatial correlations of gas and shrinkage pores ${ }^{1}$. Since the pore shapes are generated from realigned sections, there are minor losses in morphology. However, this slight simplification is generally irrelevant for the $\mathrm{FE}$ analysis. Actually, the simplifications performed in image manipulation and voxelization do retain the major features of the pores, including their high angular morphology and orientation. Figure 1 shows the 2D images of the serial sections of the aluminum die cast specimen, and the $3 \mathrm{D}$ reconstruction of a representative volume.

\section{Mesh generation}

The file containing the 3D reconstructed microstructure of the body is then exported to the mesh generation software to create appropriate FE mesh. The voxelized input in the particular case of a porous material consists of a set of $(X, Y, Z)$-coordinates of the voxel centers. For the pore contours on a given serial section, the $(\mathrm{X}, \mathrm{Y})$ -

\footnotetext{
${ }^{1}$ Bio-Research Infrastructure Construction Team, VCAD System Research Program
}

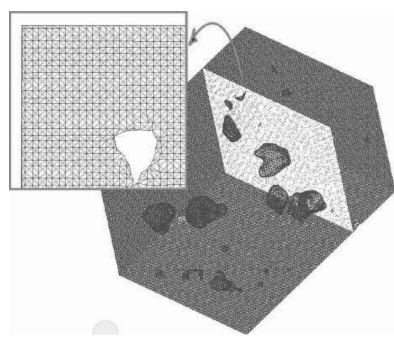

(a)

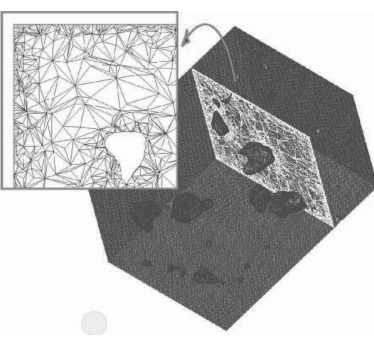

(b)
Fig. 2 Mesh generation. (a) Before simplification. (b) After simplification.

coordinates are provided by the image analyzer for closely spaced points on the pore contours, while the Z-coordinate of a voxelization equals its distance the top of the stack. Therefore, by performing image analysis on each serial section in the stack, the $(\mathrm{X}, \mathrm{Y}, \mathrm{Z})$-coordinates of points on the surfaces of all pores can be obtained, and consequently a complete 3D information on the size, shapes, orientations, and spatial arrangement of the pores in the region of interest. Utilizing the mesh generation software developed by the VCAD Modeling Team ${ }^{15)}$, initially a function for material classification is defined, by means of which the material boundaries are implicitly represented as smooth surfaces, the matrix and each individual pore being initially labeled as different materials. Then a multi-material tetrahedral mesh is generated, by using an extension of the isosurface stuffing algorithm ${ }^{16)}$. Finally, the mesh is simplified until a target number of tetrahedra is reached, under constraints related both to the mesh quality and to the accurate geometric rendering of inner and outer boundaries. An example of mesh generation and subsequent simplification, as applied to the extracted representative volume of Fig. 1, is shown in Fig. 2. As already mentioned, pores have complex shapes, they vary in size and orientations, and the surface curvature varies from one surface element to another even on the boundary of the same pore. Furthermore, the distances between pores are not uniform; some pores are very closely spaced, whereas others are not. To take into account the influence of these geometric details on the local stress and strain distributions, a very fine mesh is used near the pore surfaces and in the regions of closely spaced pores. On the other hand, for computational efficiency, it is not desirable to extend the very fine mesh over the whole volume of the region of interest, as it can be seen in Fig. 2 (b).

\section{Choice of representative volume elements}

In the study of the mechanical behavior of the heterogeneous materials, the concept of representative volume element (RVE) plays a central role. The FE 
analysis of such materials is generally based on the classical homogenization assumption : the RVE should be large enough with respect to the size of microstructural features, but small enough with respect to the wave-length of the physical fields under consideration ${ }^{17)}$. This approach is obviously justified for materials with a rather uniform distribution of the microstructural features, in other words, for macroscopically homogeneous materials. Unfortunately, the microstructure of porous cast metals does not satisfy this requirement. Actually even first-order attributes like the volume fraction of pores may have a highly heterogeneous distribution, as a result of the nonuniform temperature gradients occurring during the solidification process. In such cases, the most reasonable approach is to use the volume fraction of pores, say $\phi$. Furthermore, from a computational point of view, it is not practical to perform $\mathrm{FE}$-based simulations on very large domains containing a very large number of heterogeneities. Therefore, when calculating the effective elastic properties of porous materials, the representative volume elements should represent a good sample of the average properties and need not include the very tiny details of the microstructure. On the other hand, when investigating the failure modes of such porous materials, it is necessary to find out the most critical configurations of the microstructural features, taking into account the extent of pore clustering, the pore shapes, and even the positions of the pores with respect to the free boundaries. Whenever such second-order attributes are ignored, the experimentally determined failure criteria will inevitably display a rather large dispersion of the relevant material parameters.

In the present study, a parallelepipedic volume of about $20 \times 19 \times 15 \mathrm{~mm}^{3}$ size and average porosity of about 0.05 has been extracted from a car engine block made of an aluminum cast alloy. The 3D pore morphology has been reconstructed from the stack of 3900 serial sections ${ }^{18}$. The simulations were performed on 12 cubic subdomains of the parallelepipedic volume, having the size of $3.31 \times 1.77 \times 2.98$ $\mathrm{mm}^{3}$ and in which the porosity volume fraction varied from about $0.022 \%$ up to $25 \%$.

\section{Results and discussions}

The created FE mesh has been used for the numerical computation of the mechanical response under various loading conditions. The FE simulations have been carried out by using V-MultiMat, a software dedicated for structural analysis of multi-materials, which has been developed within the VCAD System Research Program. The following analysis is intended to illustrate the methodology used for incorporating the reconstructed 3D

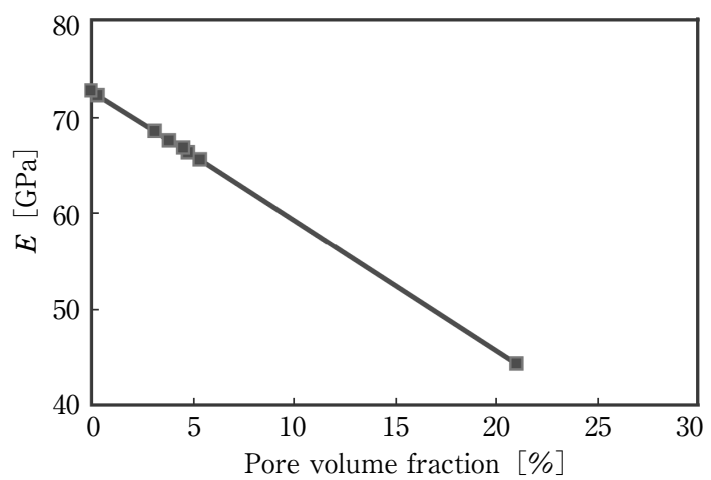

Fig. 3 Variation of the Young modulus vs. pore volume fraction. Squares: Simulation results on subdomains of different porosities. Solid line : Linear fit of the simulation results.

serial sectioning images into a computational $\mathrm{FE}$ framework, in order to correlate the porosity with the elastic properties as well as to analyze the stress and strain concentrations around the pores.

\subsection{Elasticity}

One of the most used correlations between the Young modulus $E$ and the porosity volume fraction $\phi$, is the power law $E=E_{0}\left(1-\phi / \phi_{0}\right)^{n}$ where $E_{0}$ is the Young modulus of the sound material (without pores), and $\phi_{0}$ is some critical value of $\phi$, for which the porous material can no longer sustain the loading. This power law was initially based on theoretical models of geometric stackings of solid particles with interstitial pores ${ }^{19)}$, and later substantiated by empirical data ${ }^{20)}$. Moreover, it has been suggested that the exponent $n=K_{\mathrm{t}} \phi_{0}$, where $K_{\mathrm{t}}$ is a stress concentration factor, usually larger than 1 , and related to the type and characteristics of the porosity ${ }^{21}$.

Our simulations of uniaxial compression, simple shear and volumetric compression on various RVEs of the porous aluminum die-cast have shown that the Young modulus, the bulk modulus, and the shear modulus vary almost linearly with the volume fraction of pores $\phi$, for $\phi$ less than about 0.25 . As illustrated by Fig. 3, the Young modulus $E$ changes linearly with the porosity volume fraction in the considered range of $\phi$, this result being almost independent on the pore shapes and clustering. Obviously, this linear dependence represents the linearized form of the power law, for relatively small values of $\phi$, namely $E=E_{0}\left(1-K_{\mathrm{t}} \phi\right)$. More detailed analyses and results concerning the modeling of the elastic behavior of porous casts will be presented in a separate contribution.

\subsection{Plasticity and ductile fracture}

Simulating the large deformations of a porous representative volume element allows analyzing the stress concentrations around the pores that may eventually lead to failure by fatigue or premature rupture. Figure 4 illustrates 


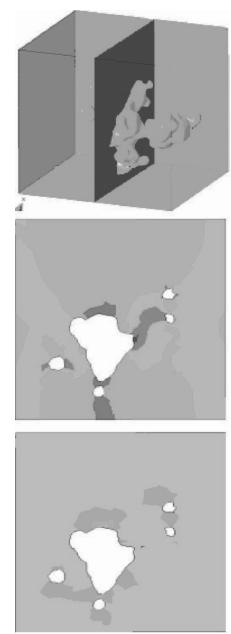

Elastic state
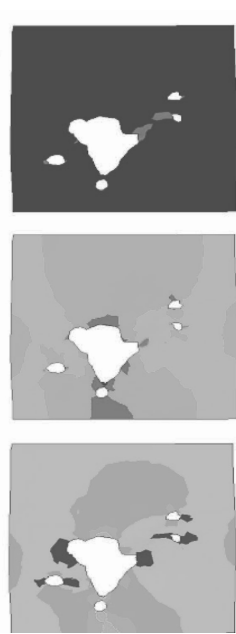

Mid-deformation
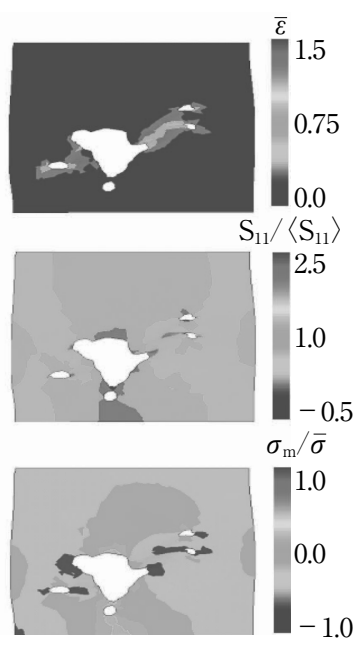

End of deformation

Fig. 4 RVE under uniaxial compression. Top: Equivalent plastic strain. Middle: Heterogeneity of the axial stress. Bottom: Stress triaxiality.

the type of results that can be obtained when simulating the large elastoplastic deformations of a porous representative volume element, by using V-MultiMat. A cubic domain of about $6.79 \mathrm{~mm}^{3}$ is subjected to uniaxial compression up to about $15 \%$ engineering strain. As expected, the simulation reveals that the distributions of local stresses and strains depend on size, orientation, and spatial arrangement of the pores. Figure 4 shows contour plots of the axial stress heterogeneity $\mathrm{S}_{11} /\left\langle\mathrm{S}_{11}\right\rangle$, of the equivalent plastic strain $\bar{\varepsilon}$, and of the stress triaxiality $\sigma_{\mathrm{m}} / \bar{\sigma}$, at different stages of deformation. Here $\mathrm{S}_{11}$ denotes the axial stress, $\left\langle\mathrm{S}_{11}\right\rangle$ is the volumetric average of $\mathrm{S}_{11}$ over the simulation domain, while $\sigma_{\mathrm{m}}$ and $\bar{\sigma}$ are the mean stress and the equivalent tensile stress, respectively. It can be seen that the plastic strain heterogeneity increases steadily with progressing deformation, eventually leading to strain localization in regions situated close to or between the pores. On the contrary, the heterogeneity of the stress triaxiality is somewhat higher in the elastic range of the deformation, but decreases to some extent at the onset of the generalized plastic flow, and remains relatively stable with progressing proportional loading. Figure 5 shows that qualitatively similar results are obtained when subjecting the porous volume to simple shear up to about $25 \%$ amount of shear.

\section{Conclusion}

The simulation results presented in this paper demonstrate that the reconstructed models adequately retain the major features of the pores, including their high angular morphology and orientation, thus capturing the relevant microstructural information for $\mathrm{FE}$-based simulations of

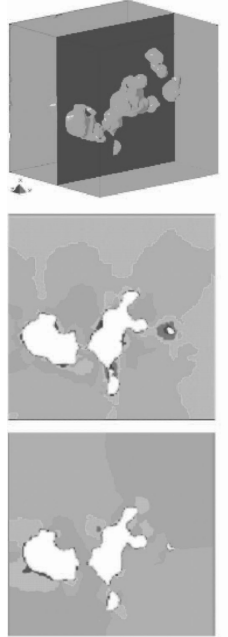

Elastic state

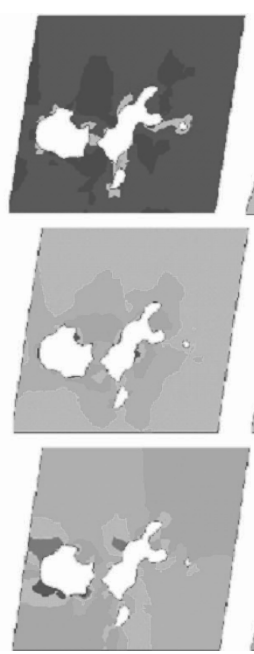

Mid-deformation

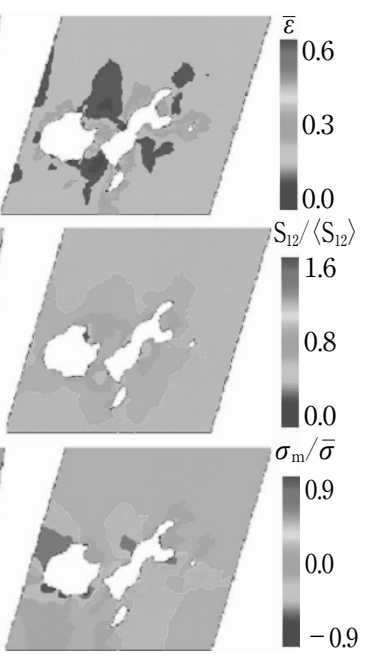

End of deformation
Fig. 5 RVE under simple shear. Top:Equivalent plastic strain. Middle: Heterogeneity of the shear stress. Bottom:Stress triaxiality.

the micromechanical response. The evaluation of the elastic stress concentrations and of their time variation is essential for both the static and fatigue analysis of the strength of porous materials in structural applications. On the other hand, the ductile fracture properties depend not only on the material, but also on the time average of some local stress measures that determine the damage accumulating with progressing deformation. In particular, recent theoretical and experimental studies (see, e.g. Ref. 23)) on the ductile fracture properties of cast aluminum alloys similar to the one considered in the present study have shown that the maximum equivalent plastic strain to fracture $\bar{\varepsilon}_{\mathrm{f}}$ depends essentially, for a given cast material, on the imposed history of stress triaxiality. In addition, a rather large spread of fracture data has been observed, which may be reasonably attributed to the presence of the pores. Clearly, the methodology presented in this paper, can provide a systematic way of realistically retaining the major features of the pore distribution, including their high angular morphology and orientation, thus capturing the relevant microstructural information for $\mathrm{FE}$-based simulations of the micromechanical response, up to the material failure by fatigue or ductile fracture.

\section{References}

1) D. Pohl and F. Redlinger: Metallographic Measurement of Pore Shape and Its Significance for the Mechanical Properties of Sintered Iron, Powder Metallurgy Int., 9, 4 (1997) 164.

2) Z. Shan and A.M. Gokhale: Micromechanics of Complex Threedimensional Microstructures, Acta Materialia, 49, 11 (2001) 2001.

3) T. Nakamura, G. Qian and C.C. Berndt: Effects of Pores on Mechanical Properties of Plasma-sprayed Ceramic Coatings, J. American Cer. Soc., 83 (2000) 578.

4) D.J. Liu : Control of Pore Geometry on Influencing the Mechanical 
Property of Porous Hydroxyapatite Bioceramic, J. Mat. Science Letters, 15, 5 (1996) 419.

5) E. Ferrie, J.Y. Buffiere and W. Ludwig : 3D Characterization of the Nucleation of a Short Fatigue Crack at a Pore in a Cast AL Alloy Using High Resolution Synchrotron Microtomography, Int. J. Fatigue, 27 (2005) 1215.

6) J. Shaocheng, G. Qi and X. Bin:Porosity Dependence of Mechanical Properties of Solid Materials, J. Mat. Science, 41, 6 (2006) 1757.

7) A. Balasundaram and A.M. Gokhale: Quantitative Characterization of Spatial Arrangement of Shrinkage and Gas (air) Pores in Cast Magnesium Alloys, Mat. Charac., 46, 5 (2001) 419.

8) R.A. Hardin and C. Beckermann: Prediction of Fatigue Life of Cast Steel in the Presence of Porosity, Proc. 61st Technical and Operating Conf., SFSA, Chicago, IL, (2007).

9) R.W. Rice: Limitations of Pore-stress Concentrations on the Mechanical Properties of Porous Materials, J. Mat. Science, 32 (1997) 4731.

10) D.A. Lados, D. Apelian and A.M. de Figueredo: Fatigue Performance of High Integrity Cast Aluminum Components, Proc. 2nd Int. Aluminum Casting Technology Symposium, (2002) 185.

11) K.M. Sigl, R.A. Hardin, R.I. Stephens and C. Beckermann : Fatigue of 8630 Cast Steel in the Presence of Porosity, Int. J. Cast Metals Research, 17 (2004) 130.

12) K. Ohura, Z. Sun, A. Makinouchi and C. Teodosiu : Volume-CAD : An Integrated Environment for Virtual Manufacturing and Structural Analysis, Adv. Mat. Research, 23 (2007) 17.

13) J.E. Spowart: Automated Serial Sectioning for 3D Analysis of
Microstructures, Scripta Materialia, 55, 1 (2006) 5.

14) N. Chawla, R.S. Sidhu and V.V. Ganesh: Three-dimensional Visualization and Microstructure-based Modeling of Deformation in Particle-reinforced Composites, Acta Materialia, 55, 6 (2006) 1541.

15) Y. Ohtake and H. Kawaharada: Tetrahedral Mesh Generation of Multi-material Solids from 3D Segmented Images, Proc. of 2007 JSPE Autumn Meeting, (2007) 323.

16) F. Labelle and J.R. Shewchuk: Isosurface Stuffing:Fast Tetrahedral Meshes with Good Dihedral Angles. ACM Transactions on Graphics (Proc. SIGGRAPH), 26, 3 (2007) 1.

17) S. Forest: Milieux Continus Généralisés et Matériaux Hétérogènes, Mines Paris, Les Presses, (2006)

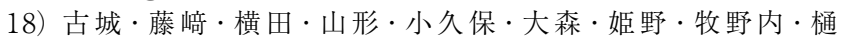
ロ：フライス切削型三次元内部構造顕微鏡を用いたアルミダイ カスト部品内の鋳巣観察, 2006 年度精密工学会秋季大会学術講 演会講演論文集，（2006/9）213.

19) R.W. Rice : Use of Normalized Porosity in Models for the Porosity Dependence of Mechanical Properties, J. Mat. Science, 40 (2005) 983.

20) C.W. Bert: Prediction of Elastic Moduli of Solids with Oriented Porosity, J. Materials Science, 20 (1985), 2220.

21) R.A. Hardin and C. Beckermann: Effect of Porosity on the Stiffness of Cast Steel, Metal. Mater. Trans. A, 38A (2007) 2992.

22) C.T. Herakovich and S.C. Baxter: Influence of Pore Geometry on the Effective Response of Porous Media, J. Mat. Science, 34 (1999) 1595.

23) H. Mae, X. Teng, Y. Bai and T. Wierzbicki : Calibration of Ductile Fracture Properties of a Cast Aluminum Alloy, Mat. Science and Eng., A459 (2007) 156. 